\title{
Three-dimensional, isotropic imaging of mouse brain using multi-view deconvolution light sheet microscopy
}

\author{
Sa Liu*, Jun Nie*, Yusha Li ${ }^{\dagger}$, Tingting $\mathrm{Yu}^{\dagger}$, Dan $\mathrm{Zhu}^{\dagger,+, \boldsymbol{\oplus}}$ and Peng Fei, ${ }^{*, \dagger, \S, \boldsymbol{\uparrow}}$ \\ *School of Optical and Electronic Information \\ Huazhong University of Science and Technology \\ Wuhan 430074, P. R. China \\ ${ }^{\dagger}$ Britton Chance Center for Biomedical Photonics \\ Wuhan National Laboratory for Optoelectronics \\ Huazhong University of Science and Technology \\ Wuhan 430074, P. R. China \\ *dawnzh@mail.hust.edu.cn \\ §feipeng@hust.edu.cn
}

Received 29 June 2017

Accepted 28 August 2017

Published 21 September 2017

\begin{abstract}
We present a three-dimensional (3D) isotropic imaging of mouse brain using light-sheet fluorescent microscopy (LSFM) in conjunction with a multi-view imaging computation. Unlike common single view LSFM is used for mouse brain imaging, the brain tissue is 3D imaged under eight views in our study, by a home-built selective plane illumination microscopy (SPIM). An output image containing complete structural information as well as significantly improved resolution ( $\sim 4$ times) are then computed based on these eight views of data, using a bead-guided multi-view registration and deconvolution. With superior imaging quality, the astrocyte and pyramidal neurons together with their subcellular nerve fibers can be clearly visualized and segmented. With further including other computational methods, this study can be potentially scaled up to map the connectome of whole mouse brain with a simple light-sheet microscope.
\end{abstract}

Keywords: Light sheet fluorescent microscopy; multi-view deconvolution; mouse brain imaging; isotropic.

\section{Introduction}

Light sheet fluorescent microscopy (LSFM) is an emerging technology that can three-dimensionally image various samples from cells to whole organisms with low phototoxicity and high speed. ${ }^{1-3}$ LSFM is fundamentally characterized by setting a separated

\footnotetext{
ๆCorresponding authors.
}

This is an Open Access article published by World Scientific Publishing Company. It is distributed under the terms of the Creative Commons Attribution 4.0 (CC-BY) License. Further distribution of this work is permitted, provided the original work is properly cited. 
plane illumination orthogonal to the wide-field detection, thus enabling optical sectioning inside the samples with sharp contrast and high axial resolution superior to conventional microscopes. ${ }^{4-7}$ In the past decade, besides the basic Gaussian light-sheet generated with a cylindrical lens, LSFM have derived a series of structural variants, including multidirectional SPIM (mSPIM), Digital Scanned Laser Light-sheet Fluorescence Microscopy (DSLM), Bessel beam plane illumination microscopy and so on. ${ }^{8-14}$ In accordance to these advanced LSFM imaging modalities, a variety of novel biomedical studies have been accomplished based on their excellent visualization. ${ }^{15-20}$

Among those applications, neural imaging of clarified mammal tissues, such as mouse brain and rat spinal cord, using LSFM tends to be a popular field that is particularly towards the high-resolution imaging under a very large field-of-view. However, LSFM is still challenging in this scenario for its axial resolution, which is typically 4 to $20 \mu \mathrm{m}$, remaining insufficient for three-dimensionally visualizing the single neurons across a large interactive area. ${ }^{21-25}$ Therefore, the fine neural connections are not able to be revealed as what have been achieved by microoptical sectioning tomography (MOST) or sequential two photon excitation microscopy. ${ }^{26-29}$ Furthermore, even for the optically-cleared organs, the light scattering as well as absorption still exists, largely deteriorating the quality from the depth of tissues. Several LSFM techniques have been developed to capture the sample images under four or more views, and computationally fuse them into single one with showing complete signals as well as improved resolution. ${ }^{30-32}$ The multi-view LSFM has been successfully applied to image live samples, ${ }^{32,33}$ such as zebrafish and Drosophila embryos, but not to the cleared brains or other tissues.

Here, we present the first brain imaging using SPIM in conjunction with multi-view deconvolution (MVD). Three-dimensional (3D) image stacks of the brain tissue are consecutively recorded under eight views, by rotating the sample a full circle. Then, a bead-based registration, followed by a multi-view Bayesian deconvolution are applied to the eight views of image, to reconstruct a complete visualization of brain cortex with an isotropic resolution $\sim 1.6 \mu \mathrm{m}$. Benefiting from the significantly improved resolution as well as more complete signals, we can segment typical neural structures, such as pyramidal neurons and astrocytes, in three dimensions, and with showing fine nerve fibers. In the following, we will briefly describe the experimental design and implementation of multi-view brain imaging, discuss how to apply the image computation, and demonstrate the isotropic visualization of brain neural connectivity.

\section{Results}

\subsection{Sample preparation}

To minimize the tissue scattering, chemical clearing of the mouse brain is an essential step before imaging. Here, we use an organic-solvent-based clearing method ( $\left.\mathrm{uDISCO}^{24}\right)$ to clarify the brain of a transgenic adult mouse that is labeled with Thy 1-green fluorescent protein (line M, Jackson Laboratory). The picture of cleared brain tissue is shown in supplementary figure S1 and compared to raw tissue.

The cleared brain is first embedded into transparent resin homogeneously mixed with fluorescent beads, to better preserve the sample fluorescence and reduce photobleaching during imaging. ${ }^{34}$ Then, the resin packed brain is mounted onto a rotating motor immersed into a glass chamber filled with refractive index-matched solvent (benzyl alcohol + benzyl benzoate + diphenyl ether, BABB-D) for upcoming LSFM imaging (Fig. 1(b)).

\subsection{Multi-view LSFM image of brain cortex}

We carry out multi-view imaging by a simple selective plane illumination light sheet microscope (SPIM) which is constructed by ourselves (Figs. 1(a) and 1(b)). The real picture of the system is shown in supplementary Fig. S2. The size of uniform illumination range of the hyperbolic laser-sheet is proportional to the thickness of laser-sheet, which can be further tuned by an adjustable slit. In our demonstration, the axial extent (thickness at beam waist) of the laser-sheet is $\sim 12 \mu \mathrm{m}$, generating a sufficiently long range to illuminate a brain cortex specimen with size around 3 by 1.5 by $1.5 \mathrm{~mm}$. A $4 \mathrm{D}$ motorized stage can move the specimen at $x-y$ plane, scan it along $z$ direction, and rotate it along $y$ direction with accurate incremental angle 


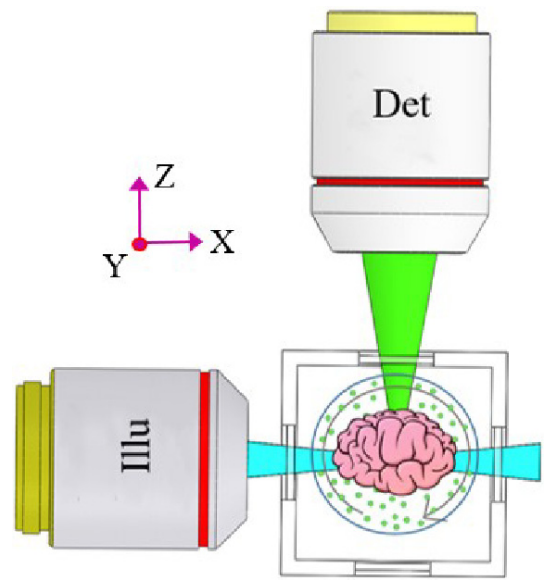

(a)

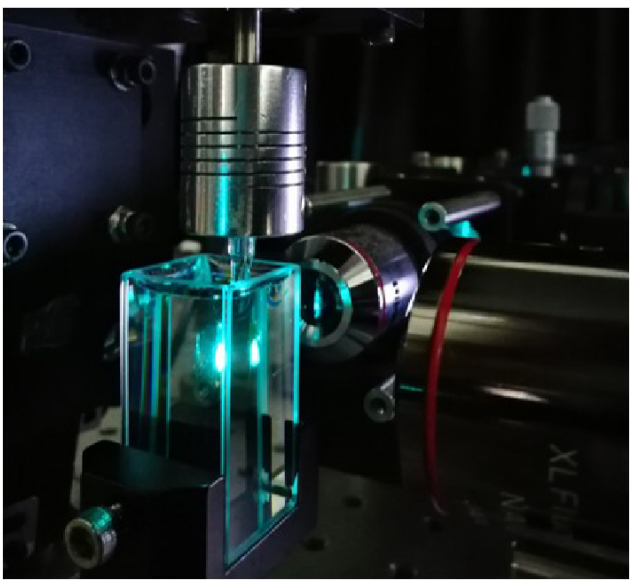

(b)

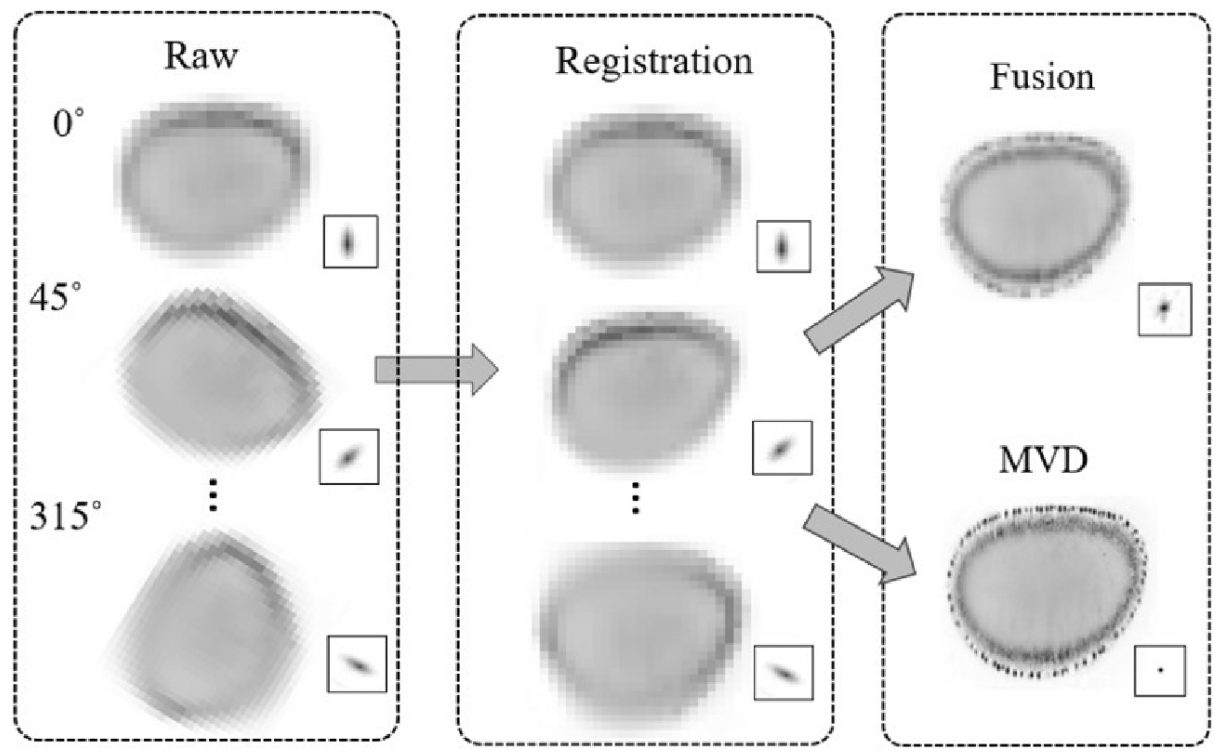

(c)

Fig. 1. Schematic of the multi-view LSFM imaging. (a) The illumination objective excites a thin plane of the brain sample using a line-focused laser-sheet. The orthogonal detection objective collects the fluorescence emission from the illuminated plane. 3D optical sectioning is accomplished by scanning the sample along $z$-axis. Through rotating the sample around $y$-axis, we then repeat the $3 \mathrm{D}$ imaging under multiple views. (b) Shows a photograph of real-world scene. (c) A brief flow chart showing the essential procedures of multi-view data computation.

(coordinates are shown in Fig. 1(a)). The planeilluminated brain is $3 \mathrm{D}$ imaged under $4 \times$ magnification with a scanning step-size $5 \mu \mathrm{m}$, yielding one view of LSFM image with voxel size 1.6 by $1.6 \mu \mathrm{m}$.

Previously, we compared the result processed by different number of views, revealing that four views MVD result does really show the isotropic resolution enhancement but the eight views deconvolution result shows higher contrast and more details in 3D space (Fig. S3). In consideration of the tradeoff between temporal and spatial resolution, eight views are the appropriate option for this experiment. Then, the sample is rotated seven times with a $45^{\circ}$ incremental angle for each view of imaging. Totally eight views of $3 \mathrm{D}$ images are recorded for the multi-view processing.

Here, we use the bead-based registration plus Bayesian deconvolution method to register, fuse and compute an output image that can finally recover the complete sample information from all the 


\section{S. Liu et al.}

views. The whole image computation procedure is further illustrated as Fig. 1(c).

\subsection{Three-dimensional visualization of the brain neurons}

We extract the sub-resolution beads $(\sim 1 \mu \mathrm{m}$ in diameter) from the images to characterize the multiview imaging performance. Figure 2 shows the resolved beads, which can be treated as system point spread function, in raw image (reference view, Fig. 2(a)), average fused image (Fig. 2(b)), and the final multi-view deconvolution image (Fig. 2(c)). As shown by comparison, the resolution enhancement by multi-view processing is quite obvious, especially along the axial direction ( $y z, x z$ planes). After Bayesian deconvolution, the fusion artifacts (shown in Fig. 2(b)) further disappear with finally showing a size-reduced, isotropically-resolved sphere.
Then, we three-dimensionally visualize the brain images in Fig. 3. Three volumes containing astrocyte (I), dense pyramidal neuron (II), and sparse pyramidal neurons (III) are selected from the complete brain sample (Fig. 3(a)), and shown as magnified views of Figs. 3(b)-3(d), respectively. In contrast to the poorly-resolved neuron sub-structures in the raw single-view image (Figs. 3(b)-3(d), left), MVD image clearly shows the patterns of dendrites and axons (Figs. 3(b)-3(d), right). The achieved resolving power of MVD image originally taken by $4 \times$ objective plus $12 \mu \mathrm{m}$ laser-sheet is even superior to the raw LSFM image taken by a $10 \times$ objective plus $6 \mu \mathrm{m}$ laser-sheet (Fig. 3(b), middle). With high-quality, $3 \mathrm{D}$ visualization, we can further segment single neurons with displaying their subcellular nerve fibers (Fig. 3(e)-I and III). Meanwhile, two adjacent pyramidal neurons can be also separated from each other by the virtue of improved resolution (Fig. 3(e)-II).

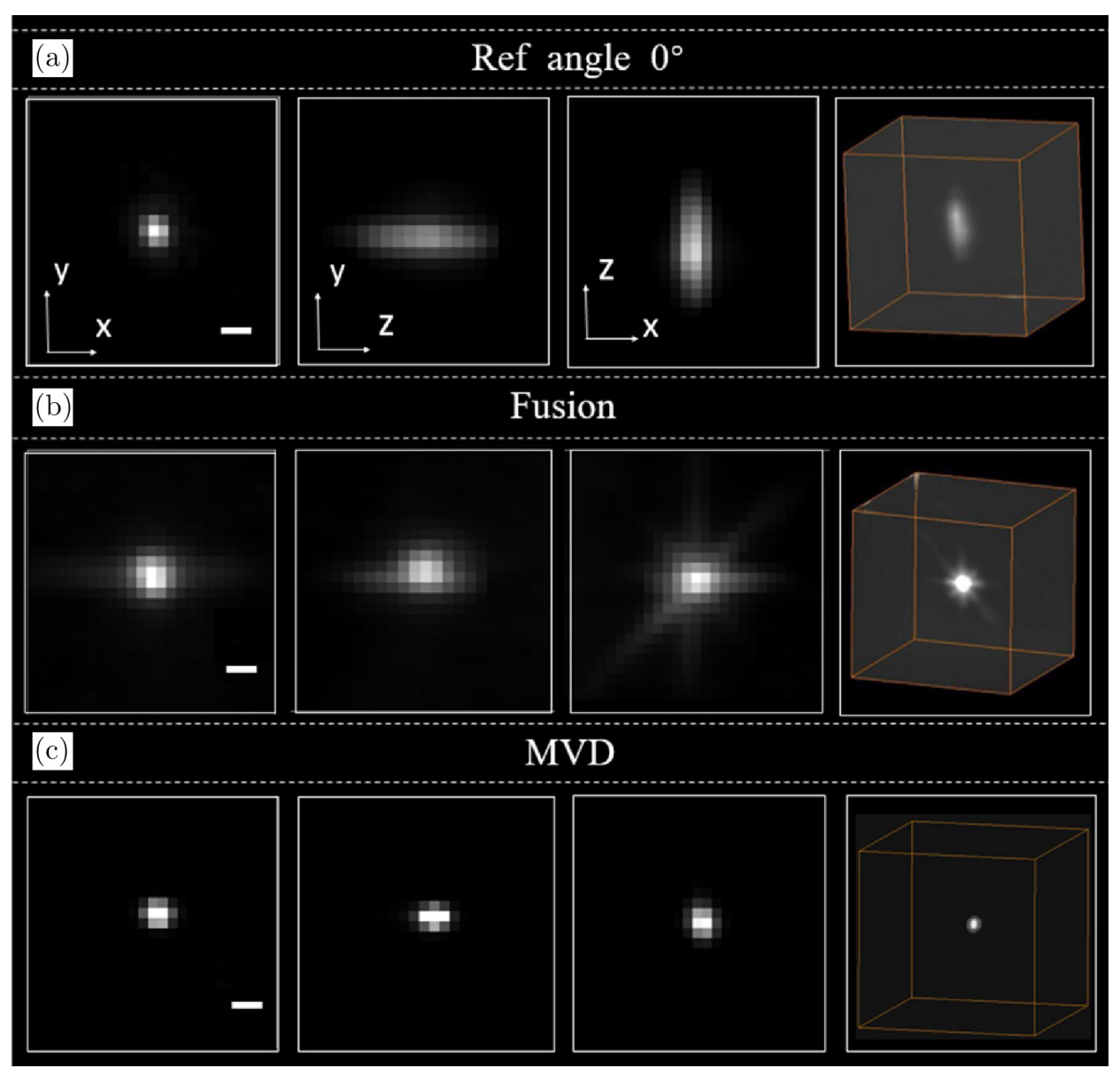

Fig. 2. Characterization using fluorescent bead. (a)-(c) compare the fluorescent beads $(\sim 1 \mu \mathrm{m})$ resolved by single-view LSFM $\left(\right.$ angle $\left.0^{\circ}\right)$. 8-view fusion and 8-view Bayesian deconvolution, respectively. The $x-y, y-z, x-z$ planes and 3D rendering of the bead are shown from the left to the right. Scale bar is $5 \mu \mathrm{m}$. 

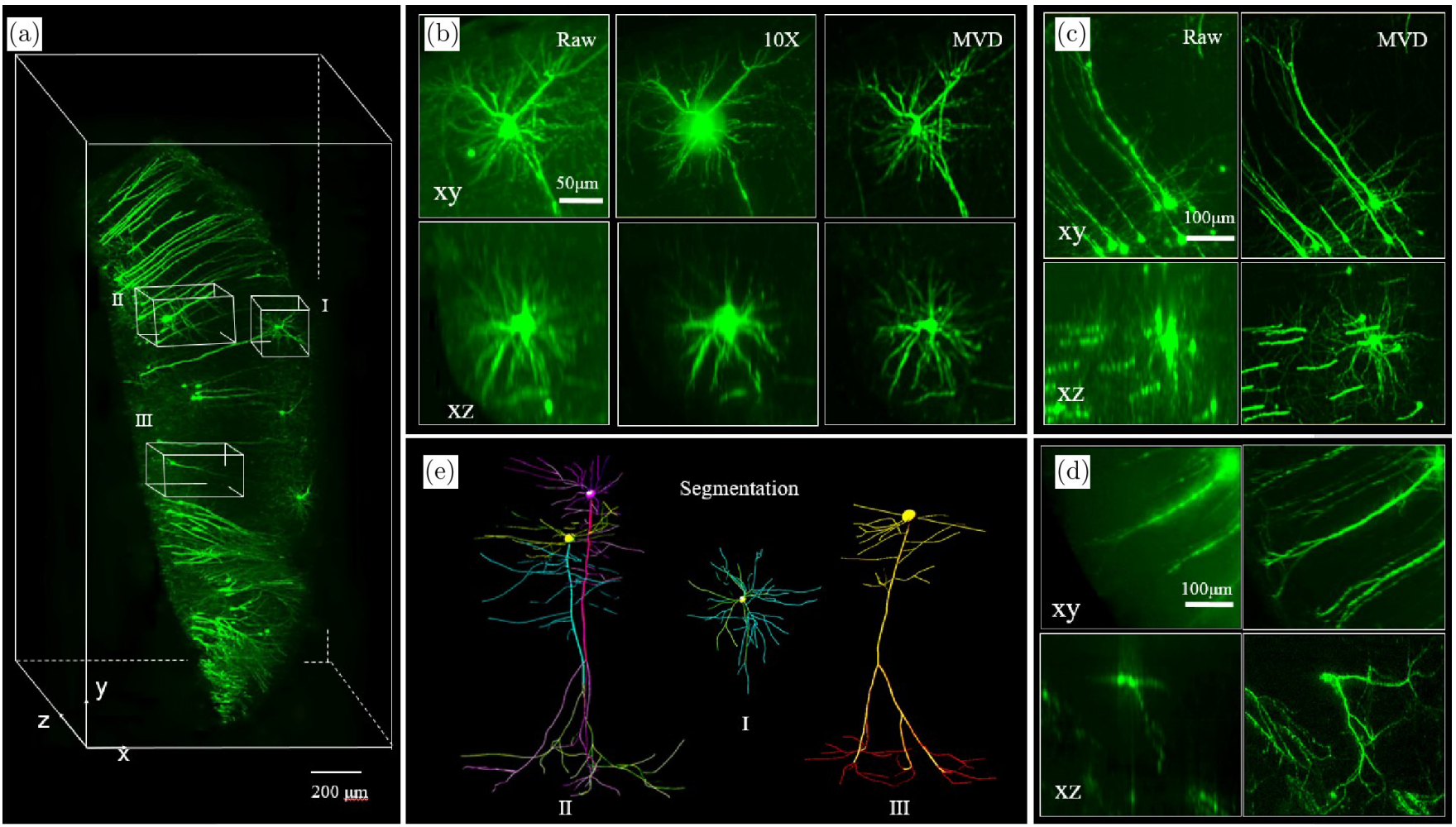

Fig. 3. Isotropic visualization of brain cortex by multi-view LSFM. (a) The 3D rendering of the cleared brain cortex tissue. The magnified views of three selected regions, I-III, are shown as (b)-(d), respectively. The maximum-intensity-projections (MIPs) of $x y$ and $y z$ planes of the selected areas are compared by three imaging modes, which are raw single view image (b, left), highmagnification single view image (middle) and MVD image (right). The multi-view deconvolution result demonstrates best visualizations with showing an improved isotropic resolution. (e) Image segmentation of single astrocyte, single pyramidal neuron and two adjacent pyramidal neurons, corresponding to the regions (I) to (III), respectively.

\section{Conclusion}

In this work, we use multi-view light-sheet imaging for the first time to achieve the isotropic imaging of mouse brain at cellular resolution. MVD improves the optical sectioning performance with circumventing the use of high NA, high-maintenance optics at the same time. Thus, it is much less vulnerable to spherical aberration which often causes noticeable deterioration in deep tissue imaging. Compared to the regular single-view LSFM imaging of mouse brain, our method recovers more details, allowing clear segmentation of single neurons. The multi-view deconvolution also represents a cost-effective strategy for brain imaging, without increasing the current LSFM system's cost and complexity. This underlying robustness allows MVD-LSFM prototype to maintain a simple set-up as well as superior performance for large sample imaging. Furthermore, through combining this multi-view approach with other super resolution techniques, a large volume and high-resolution imaging of whole mouse brain can be expected to map out the complete brain neural network, unraveling the long distance projection that cross over different encephalic regions. Our demonstration makes an initial study towards mouse brain-wide connectome by a simple light-sheet microscope, which can be readily accessed in even resource- limited environments, such as a less-funded biology laboratory. It thereby shows good potential on benefiting the neuroscience, histopathology and other tissue/organ scale studies.

\section{Acknowledgments}

This research has received funding support from 1000 Youth Talents Plan of China (P.F.), Fundamental Research Program of Shenzhen (P.F., JCYJ20160429182424047), National Science Foundation of China (NSFC31571002,D.Z), Graduates' Innovation Fund of Huazhong University of Science and Technology (5003182004). 


\section{References}

1. J. Huisken, D. Y. Stainier, "Selective plane illumination microscopy techniques in developmental biology", Development 136, 1963-1975 (2009).

2. P. J. Keller, M. B. Ahrens, J. Freeman, "Light-sheet imaging for systems neuroscience", Nat Methods 12, 27-29 (2015).

3. P. J. Keller, M. B. Ahrens, "Visualizing whole-brain activity and development at the single-cell level using light-sheet microscopy", Neuron 85, 462-483 (2015), doi: 10.1016/j.neuron.2014.12.039.

4. K. Becker, N. Jahrling, E. R. Kramer, F. Schnorrer, H. U. Dodt, "Ultramicroscopy: 3D reconstruction of large microscopical specimens", J. Biophoton. 1, 36-42 (2008), doi: 10.1002/jbio.200710011.

5. P. A. Santi et al., "Thin-sheet laser imaging microscopy for optical sectioning of thick tissues", BioTechniques 46, 287-294 (2009), doi: 10.2144/ 000113087.

6. P. G. Pitrone et al., "OpenSPIM: An open-access light-sheet microscopy platform", Nat. Methods 10, 598-599 (2013).

7. Z. Guan et al., "Compact plane illumination plugin device to enable light sheet fluorescence imaging of multi-cellular organisms on an inverted wide-field microscope", Biomed. Opt. Express 7, 194-208 (2016).

8. J. Huisken, J. Swoger, F. Del Bene, J. Wittbrodt, E. H. Stelzer, "Optical sectioning deep inside live embryos by selective plane illumination microscopy", Science 305, 1007-1009 (2004).

9. J. Huisken, D. Y. Stainier, "Even fluorescence excitation by multidirectional selective plane illumination microscopy (mSPIM)", Opt. Lett. 32, 2608-2610 (2007).

10. P. J. Keller et al., "Fast, high-contrast imaging of animal development with scanneds light sheet-based structured-illumination microscopy", Nat. Methods 7, 637-642 (2010), doi: 10.1038/nmeth.1476.

11. P. J. Keller, A. D. Schmidt, J. Wittbrodt, E. H. Stelzer, "Reconstruction of zebrafish early embryonic development by scanned light sheet microscopy", Science 322, 1065-1069 (2008).

12. R. Tomer, K. Khairy, F. Amat, P. J. Keller, "Quantitative high-speed imaging of entire developing embryos with simultaneous multiview lightsheet microscopy", Nat. Methods 9, 755-763 (2012).

13. T. A. Planchon et al., "Rapid three-dimensional isotropic imaging of living cells using Bessel beam plane illumination", Nat. Methods 8, 417-423 (2011), doi: 10.1038/nmeth.1586.

14. B. C. Chen et al., "Lattice light-sheet microscopy: Imaging molecules to embryos at high spatiotemporal resolution", Science 346, 1257998 (2014), doi: $10.1126 /$ science.1257998.
15. R. Arnaout et al., "Zebrafish model for human long QT syndrome", Proc. Natl. Acad. Sci. USA 104, 11316-11321 (2007), doi: 10.1073/pnas.0702724104.

16. B. Schmid et al., "High-speed panoramic light-sheet microscopy reveals global endodermal cell dynamics", Nat. Commun. 4, 2207 (2013), doi: $10.1038 /$ ncomms 3207 .

17. T. F. Holekamp, D. Turaga, T. E. Holy, "Fast threedimensional fluorescence imaging of activity in neural populations by objective-coupled planar illumination microscopy", Neuron 57, 661-672 (2008), doi: 10.1016/j.neuron.2008.01.011.

18. J. Lee et al., "4-dimensional light-sheet microscopy to elucidate shear stress modulation of cardiac trabeculation", J. Clin. Invest. 126, 1679 (2016).

19. M. Mickoleit et al., "High-resolution reconstruction of the beating zebrafish heart", Nat. Methods 11, 919-922 (2014).

20. F. Pampaloni, N. Ansari, E. H. Stelzer, "High-resolution deep imaging of live cellular spheroids with light-sheet-based fluorescence microscopy", Cell Tissue Res. 352, 161-177 (2013).

21. H. U. Dodt et al., "Ultramicroscopy: Three-dimensional visualization of neuronal networks in the whole mouse brain", Nat. Methods 4, 331-336 (2007), doi: 10.1038/nmeth1036.

22. R. Tomer, L. Ye, B. Hsueh, K. Deisseroth, "Advanced CLARITY for rapid and high-resolution imaging of intact tissues", Nat. Protocols 9, 16821697 (2014).

23. E. A. Susaki et al., "Advanced CUBIC protocols for whole-brain and whole-body clearing and imaging", Nat. Protocols 10, 1709-1727 (2015).

24. C. Pan et al., "Shrinkage-mediated imaging of entire organs and organisms using uDISCO", Nat. Methods (2016).

25. E. A. Susaki et al., "Whole-brain imaging with single-cell resolution using chemical cocktails and computational analysis", Cell 157, 726-739 (2014).

26. T. Quan et al., "NeuroGPS-Tree: automatic reconstruction of large-scale neuronal populations with dense neurites", Nat. Methods 13, 12142 (2016).

27. H. Gong et al., "High-throughput dual-colour precision imaging for brain-wide connectome with cytoarchitectonic landmarks at the cellular level", Nat. Commun. 7 (2016).

28. F. Helmchen, W. Denk, "Deep tissue two-photon microscopy", Nat. Methods 2, 932-940 (2005).

29. N. G. Horton et al., "In vivo three-photon microscopy of subcortical structures within an intact mouse brain", Nat. Photon. 7, 205-209 (2013).

30. J. Swoger, P. Verveer, K. Greger, J. Huisken, E. H. Stelzer, "Multi-view image fusion improves resolution in three-dimensional microscopy", Opt. Express 15, 8029-8042 (2007). 
31. S. Preibisch, S. Saalfeld, J. Schindelin, P. Tomancak, "Software for bead-based registration of selective plane illumination microscopy data", Nat. Methods 7, 418-419 (2010).

32. S. Preibisch et al., "Efficient Bayesian-based multiview deconvolution", Nat. Methods 11, 645-648 (2014).

33. M. B. Ahrens, M. B. Orger, D. N. Robson, J. M. Li, P. J. Keller, "Whole-brain functional imaging at cellular resolution using light-sheet microscopy", Nat. Methods 10, 413-420 (2013), doi: 10.1038/ nmeth.2434.

34. K. Becker et al., "Reduction of photo bleaching and long term archiving of chemically cleared GFPexpressing mouse brains", PloS one 9, e114149 (2014). 